LBNL-39093

$\mathrm{UC}-414$

CBP Note - 185

\title{
Gamma-Gamma Colliders
}

\author{
Kwang-Je Kim and Andrew Sessler
}

Center for Beam Physics

Accelerator and Fusion Research Division

Ernest Orlando Lawrence Berkeley National Laboratory

University of California

Berkeley, California 94720

June 1996

This work was supported by the Director, Office of Energy Research, Office of High Energy and Nuclear Physics, Division of High Energy Physics, of the U.S. Department of Energy under Contract No. DE-AC0376 SF00098.

RTMTIMON OF THIS DOCUMENT IS UNLLATED. 
DISCLAIMER

\section{Portions of this document may be illegible in electronic image products. Images are produced from the best available original document.}

\section{DISCLAIMER}

This report was prepared as an account of work sponsored by an agency of the United States Government. Neither the United States Government nor any agency thereof, nor any of their employees, makes any warranty, express or implied, or assumes any legal liability or responsibility for the accuracy, completeness, or usefulness of any information, apparatus, product, or process disclosed, or represents that its use would not infringe privately owned rights. Reference herein to any specific commercial product, process, or service by trade name, trademark, manufacturer, or otherwise does not necessarily constitute or imply its endorsement, recommendation, or favoring by the United States Government or any agency thereof. The views and opinions of authors expressed herein do not necessarily state or reflect those of the United States Government or any agency thereof. 


\title{
Gamma-Gamma Colliders
}

\author{
by Kwang-Je Kim and Andrew Sessler
}

Photon beams can be made so energetic and so intense that when brought into collision with each other they can produce copious amounts of elementary particles.

Almost everyone knows that light doesn't effect light. Suppose you stand near the corner of a darkened room with a flashlight in each hand and you shine the right hand beam on the left wall and the left hand beam through the other beam onto the right hand wall. Then if you move one flashlight up and down its spot on the wall will move up and down, but it will have no effect on the other beam. All this was described very elegantly in 1864 by James Maxwell who made the electrodynamic equations linear.

However, as the flashlights are made brighter and brighter, one beam does in fact begin to effect the other. The effect is the scattering of light on light and it is purely a quantum mechanical effect; it wouldn't happen classically. Now suppose the flashlight beam is made more energetic by increasing the frequency of the light so that it becomes more blue, then passes into the ultra-violet, becomes $x$-rays, and even gamma rays. At first quantum electrodynamic effects occu,r and pairs of electrons and positrons are produced, soon quantum chromodynamic effects occur, and strange particles are produced. Once again, common knowledge is not accurate knowledge.

Gamma-gamma colliders are all about what we just described; namely, making intense beams of gamma rays and having them collide so as to make elementary particles. We shall show, in this article, that constructing a gamma-gamma collider as an add-on to a electron-positron linear collider is possible with present technology and that it does not require much additional cost. Furthermore, we shall show that the resulting capability is very interesting from a particle physics point of view. An overview of a linear collider, with a second interaction region devoted to $\gamma$ collisions is shown in Figure 1 below.

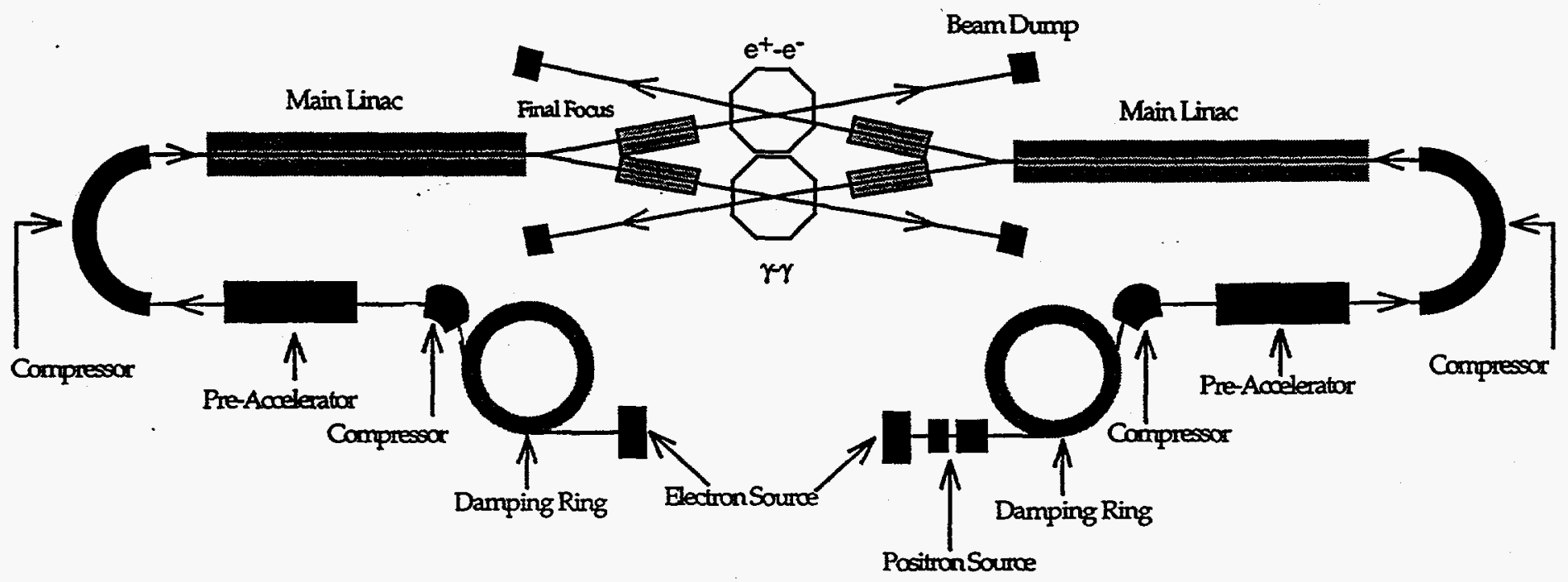

Figure 1. A Linear Collider with a second interaction region devoted to $\gamma-\gamma$ collisions. 


\section{Producing the Gamma Rays}

Perhap first of all, we should describe how intense gamma rays can be made. The best manner is by Compton back-scattering of almost visible photons from an intense, high energy, electron beam. A diagram of the creation of gamma rays in the so-called conversion region is shown in Figure 2. The resulting gamma rays will have a spectrum that extends up to approximately $80 \%$ of the electron energy. The intensity can be adequately high if the incoming beam of photons is adequately intense. How intense must it be and how intense can it be?

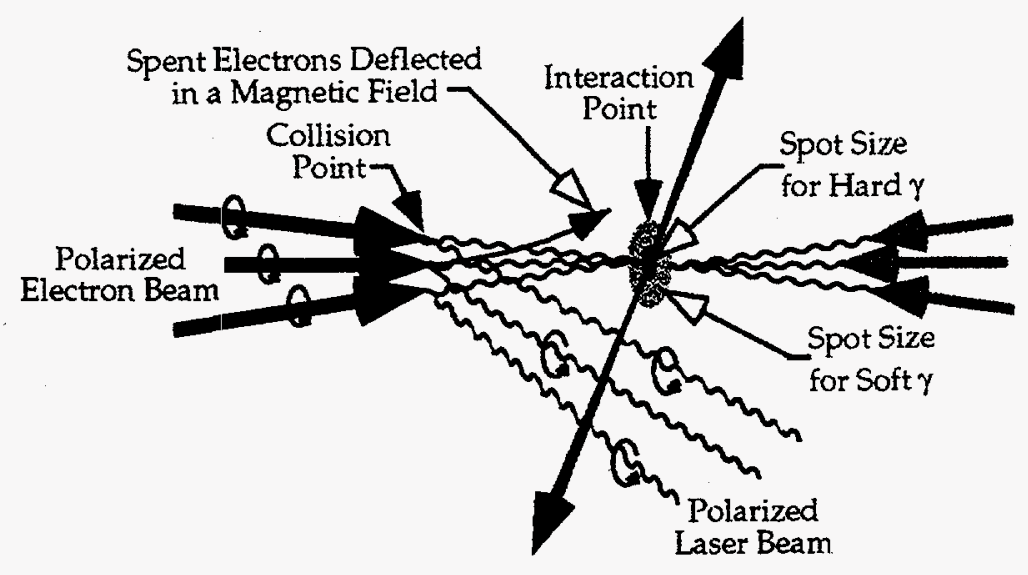

Figure 2. A sketch showing the conversion region where laser photons are converted by Compton back-scattering into high energy gamma photons.

Since the process is going to be an add-on to a linear collider we have a measure of acceptability at hand; namely, the luminosity of the $\mathrm{e}^{+} \mathrm{e}^{-}$collider. To match the $\mathrm{e}^{+} \mathrm{e}^{-}$luminosity in a $\gamma$ collider requires that we produce one gamma ray from each electron. That is just correct energetically, since in colliding with a photon the electron transfers essentially all of its energy to the photon and is, therefore, not available for the creation of a second gamma ray. Now we must ask how many photons do we need to collide with the electron beam so as to make one gamma ray from each electron? The cross section for Compton back-scattering is approximately the Thomson cross section; namely $(8 / 3) \pi r_{0}{ }^{2}$, where $r_{0}$ is the classical electron radius. Combining this with the cross sectional area of the laser pulse, we can deduce that about $10^{9}$ photons need to be collided with each electron to make one photon backscattered and thus to make one gamma ray. For a typical collider bunch of $10^{10}$ electrons we need $10^{19}$ photons. The mathematics to go with this discussion and, in particular a discussion of polarization phenomena, may be found in the sidebar on the next page. If the incident photon intensity is too large, there will be undesirable non-linear quantum electrodynamic effects. These can simply be avoided by making the conversion region longer.

Now, the incident photon can not be too energetic or else the photons in the incident photon stream can interact with the back-scattered gamma rays and produce electron pairs. It is easy to see that the criterion for the absence of this process is that the wavelength of incident photons must be longer than $3.93 \mathrm{E}[\mathrm{TeV}]$, where $\mathrm{E}$ is the energy of one (of the two) electron beams. Furthermore, one should be close to the limit. Thus for a $250 \mathrm{GeV} \times 250 \mathrm{GeV}$ collider, the photon wavelength should be about 1 micron. Now a 1 micron photon has an energy of $1 \mathrm{eV}$ and thus, although $10^{19}$ photons is a lot of photons, it is only about 1 joule of energy. A laser able to produce an energy of 1 joule is not excessive at all; in fact such lasers are rather commonplace. 
Note that the high luminosity of an $\mathrm{e}^{+} \mathrm{e}^{-}$ collider is the result of the very small spot size at the collision point: typically tens of nanometers. Note also that the conversion point at which the Compton back-scattering takes place can't be too far from the $\mathrm{e}^{+} \mathrm{e}^{-}$collision point or else the natural spreading angle of the produced gamma rays, of order $1 / \gamma$, will diffuse the collision point too much. On the other hand, the greater the distance between the conversion point and the collision point the more monochromatic will be the gamma ray spectrum. For typical colliders the conversion point will be less than $1 \mathrm{~cm}$ from the collision point.

Nevertheless, at the conversion point the electron beam is much wider than it is at the crossing point; perhaps even 100 times wider. Thus gamma rays produced at the conversion point would seem to have a radial extent when they reach the crossing point. If that were the case the luminosity of a $\gamma$ collider would be very much reduced from that of an $e^{+} e^{-}$collider. But that isn't the case! The gamma rays, produced at the conversion point, are" focused" to the spot the electrons would have occupied at the collision point. Everyone knows you can't focus gamma rays, but you can produce them to be focused, and that is precisely what is done. This happens automatically, for the electrons have much more momentum than the laser photon and the produced gamma ray proceeds along the direction of the electron. Thus, to make a $\gamma$ collider, it is only necessary to focus the electrons which is just what is normally done in $\mathrm{e}^{+} \mathrm{e}^{-}$colliders.

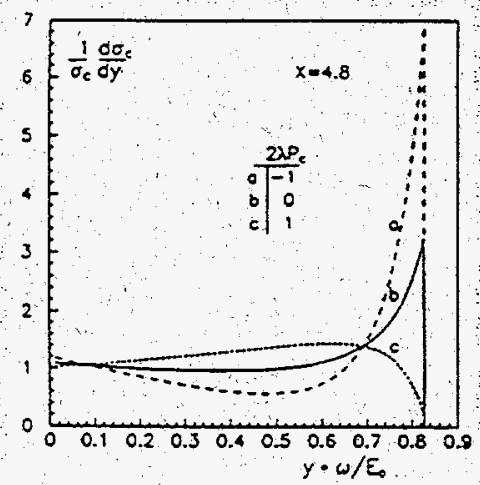

$+$

The spectrum of the Compton scattered photons for different conibinations of helicities (see text for further explanation).

A measure of the energy of the colliding electron-photon system in Compton scattering is the invarient quantity $x=12.3 \mathrm{E}[\mathrm{TeV}] /$ $\lambda$ [microns], where $\lambda$ is the wavelength of the laser photon. The value of $x$ should be smaller than 4.83 , otherwise the high energy gamma photons produced by the Compton backscattering have a good chance to disappear by colliding with other laser photons producing $e^{+} e^{-}$ pairs (the Breit-Wheeler process) On the other hand, the maximum energy of the back-scattered gamma photon is $(x /(1+x))$ E. For a gamma-gamma collider $x$ is chosen to be about 4.8 in order to produce gamma photons with as high as energy as possible while avoiding the pair production threshold It then follows that the maximum photon energy is about $80 \%$ of the electron enery, and that the optimum laser wavelength for $E=250 \mathrm{GeV}$ is about $1 \mathrm{mi}$ cron:

The cross section for the Compton process a, ranges from the classical Thomson cross section $(8 / 3) \mathrm{xO}^{2}$ for $x$ much smaller than unity to about $\pi \mathrm{ro}^{2}=25 \times 10^{-25} \mathrm{~cm}^{2}$ at $x=4.8$. The illustration below left shows the differential Comption scattering as a function of $y=$ the energy of the backscattered gamma-photon/E. We notice the marked difference in the y-dependence on the polarization state when the helicity of the electrons is opposite to that of the laser photons, the differential cooss section shown by curvea is sharply peaked on the narrow negion around the rnaximum value of $y$. The peak is less promi nent for the unpolarized case shown by curve $b$, and disappears altogether when the helicities are parallelas shown by curve $c$ for a gammagamma collide, it is clearly most ad vantageous to choose casea

To produce one gamina photon per electron, the laser photons, each with an effective cross section $\sigma_{\mathcal{O}}$ must cover the full transverse area of the laser pulse $A$, ocNlaser $=A$, where Nlaser is the total number of laser photons in the pulse $\mathrm{On}$ the other hand, $\mathrm{A}=\mathrm{A}$ because of diffraction, where I s the effective length of the laser pulse which in tarm is about the same as the electron pulse length, Taking the pulse length to be about $1 \mathrm{ps}$ and using the laser wavelength and the Compton cooss section discussed above, it follows that the Nlaser $-10^{19}$

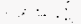


Further discussion of the parameters for a design of a gamma-gamma collider as a second interaction region of the Next Linear Collider (NLC) is presented in a sidebar on the next page. This is in a sense a minimal design, because the electron beam parameters optimized for $\mathrm{e}^{+} \mathrm{e}^{-}$collisions before the final focus system are taken as they are. The only modifications are in the design of the final focus optics and an adequate laser for Compton conversion. With this approach, the luminosity for the gamma-gamma collisions, within $20 \%$ bandwidth, is about $10 \%$ of that in the $e^{+} e^{-}$collider mode. This is sufficient to carry out interesting physics experiments. In particular the cross sections for some reactions are larger at a gamma collider than at a conventional electron collider. Clearly, what is needed is a careful discussion of expected reaction rates, and background rates, for it is after all the signal to noise that is the relevant thing. If the electron beam is modified, for example by changing the damping ring so as to make an electron beam more favorable for a gamma-gamma collider (for example with a higher bunch charge), then the luminosity may be increased to a value comparable or, according to some speculations, even higher than that of an electron-positron collider, because beam-beam effects are less severe for gamma-gamma collisions.

\section{The Particle Physics}

A discussion of the laser which provides the photons for a gamma collider will be given later, coupled with some discussions of the requisite optics in $\gamma$ colliders. Here we comment upon the particle physics opportunities that such a device would provide. One of the most interesting physics programs is measurement of the Higgs boson partial width into $\gamma$. This partial width is sensitive to physics beyond the Standard Model. A further discussion of the Higgs width is presented in a sidebar on page 6.

Another interesting set of reactions is the decay of the Higgs into either to bb or to ZZ. One could also study the $\mathrm{CP}$ eigenvalue of the Higgs by means of polarized gamma rays. It is important to note that in a $\gamma /$ collider one can use the full center-o- mass energy to produce Higgs bosons in the s-channel, where as one would need to produce them in pairs at an $\mathrm{e}^{+} \mathrm{e}^{-}$ collider. Thus the energy reach of a $\gamma$ collider is much increased over that of an $\mathrm{e}^{+} \mathrm{e}^{-}$collider.

If supersymmetry exists, charged super particles can be produced at a $\gamma /$ collider with reasonable cross sections. The main source of background is WW pairs. Finally, if some of the particles in the Standard Model are composites, made of more fundamental particles, they should either have excited states decaying into the ground state by emission of $\gamma$ or $Z$ particles, or anomalous interactions as low-energy limits of their form factors. If the W-boson is composite it may have an anomalous magnetic moment (or electric quadrupole moment) and either of these should be measurable given the copious production of $\mathrm{W}$ expected at a $\gamma \gamma$ collider.

\section{The LASER AND ITs OPTICS}

Returning now to the technical problems associated with constructing a gamma collider, we need to discuss the laser optics in the conversion/interaction region. As the reader will appreciate, the region is very crowded. Furthermore, for detector reasonsit is important that a minimum amount of mass is put into this region. The simplest configuration, and probably the one that will be used in practice is to bring the opposing laser beam for each beam of electrons, in from the opposite side, and after conversion dispose of (the essentially unaltered) laser beam.

In devising suitable optics for the intense laser beam we must consider a number of elements. Firstly, transmissive optics are for the most part, not feasible. Secondly, the overlap between the electron and photon beam must be good, that is, the two axes must be closely 


\section{PARAMETERS FOR A GAMMA-GAMMA COLLIDER AS A SECOND INTERACTION REGION FOR THE NEXT LINEAR COLLIDER}

The table below shows the main parameters of a gamma-gamma collider as the second interaction region of the NLC. The electron beam parameters up to the final focus system are taken to be the same as that for the first interaction region (forete collisions). The horizontal and the vertical beam sizes in the case of the $\mathrm{e}^{+} \mathrm{e}$ collisions are designed to be as small as possible within the constraints of the beam beam effects. In the case of the $\gamma$ collisions, the electron beam sizes at the interaction point (assuming the electrons are allowed to collide without being Comption scattered) are determined from different considerations. The vertical beam size should not be smaller than that determined by the $1 / \gamma$ spreading of the gamma rays between the conversion point and the interaction point. The horizontal beam size should be made as small as possible and consistent with various lattice design constraints of the final focus system (such as chromaticity control, beam blow-up caused by synchrotron radiation in the final quadrupoles known as the Oide effects, etc.) A reasonable choice appears to be that the beta functions in the horizontal and the vertical directions have the same values, $0.5 \mathrm{~mm}$. This leads to a geometric electron-electron luminosity (again assuming the electrons are allowed to collide without being Compton scattered) of about $1 \times 10^{34} / \mathrm{cm}^{2} / \mathrm{s}$, which is about twice that of the $e^{+} e$ collisions.

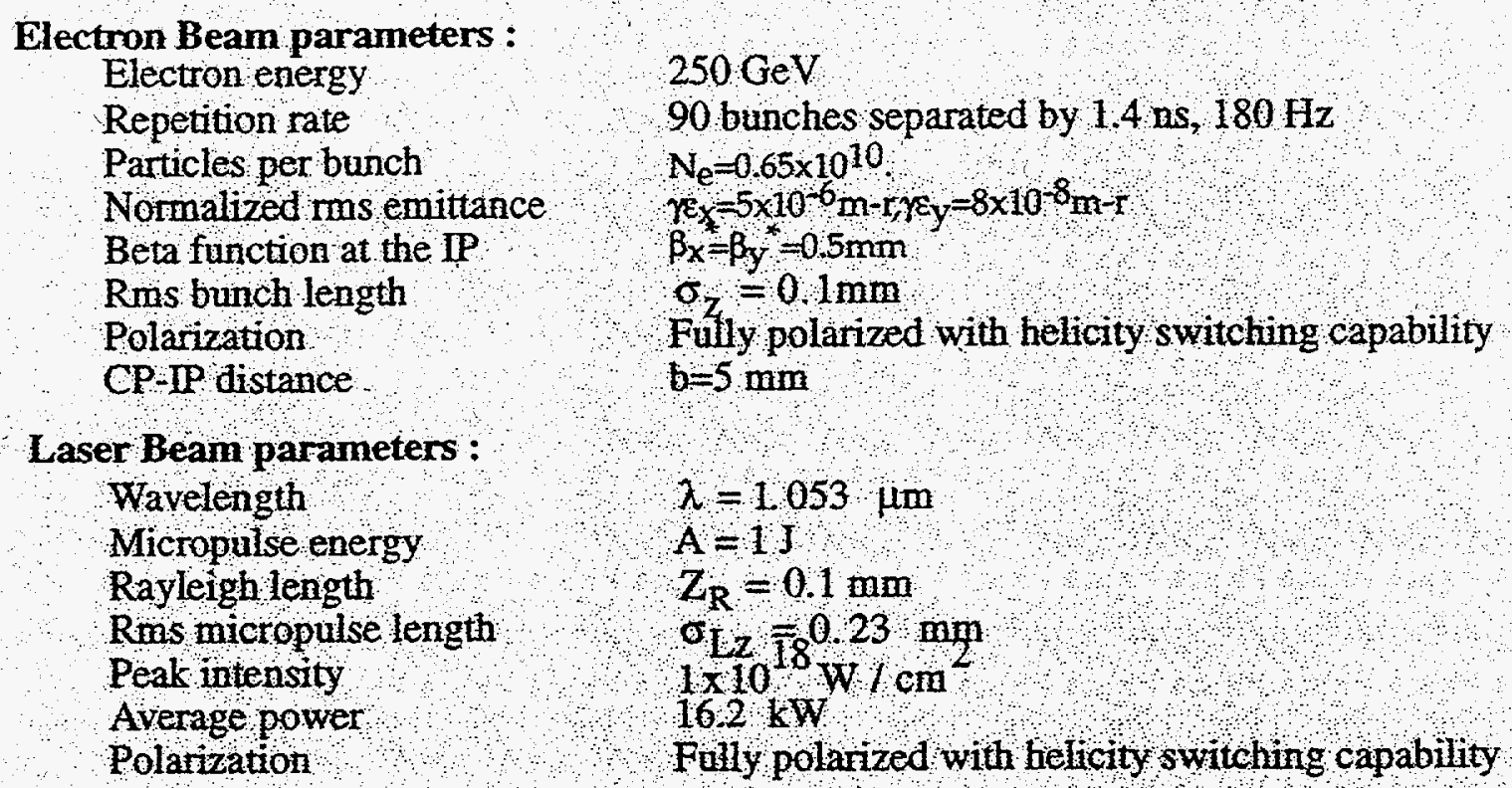

As noted before, the laser parameters are chosen to optimize the conversion of the electron beams into the high energy photon beams. With 1 joule per pulse foetsed to $1 \times 10^{18} \mathrm{~W} / \mathrm{cm}^{2}$, the probability of producing a high energy gamma photon per electron (the conversion efficiency) is about $65 \%$. Therefore the luninosity of the $\gamma$ collisions will be about $(0.65) 2=0.43$ times the geometric electron-electron luminosity However, the spectrum of the gamma-ray photons is fairly wide, with a peak near $83 \%$ of the electron energy. The luminosity near this peak, within a $20 \%$ bandwidth, is about $10 \%$ of the geometric luminosity, that is, about $1 \times 10^{33} / \mathrm{cm}^{2} / \mathrm{s}$. 
aligned and the Rayleigh length must be adequately long. Because of the high peak and average power, the spent laser beam must be transported to an external beam dump. Because the two electron beams intersect at a small angle (so that the spent electron beam has a separate channel to exit the detector) the used laser beam from one side will intersect the optics of the other side. Since two pulses will fall on the same mirror, it is necessary to locate mirrors at points where two pulses will not reach them at the same time. A possible mirror arrangement to bring the laser pulses to the conversion point and to dispose of them in the tight space limited by the vertex chamber, the mask, and quadrupoles is shown on page 7 .

Finally, we must address the question of developing a laser for the purposes of a $\gamma$ collider. The requirements listed in the box on page 5 are a pulse of about 1 joule, a pulse length of a few picoseconds (corresponding to a peak power of a few terrawatts), an average power of 16 kilowatts (corresponding to about 100 bunches per pulse and a rep-rate of about $160 \mathrm{~Hz}$ ), and variable polarization. Using the chirped pulse amplification technique, solid-state lasers already give peak powers that satisfy our requirements; they only fail our needs in that the average power is currently in the few watt range. Two critical technolgies for constructing high average power lasers for a gamma-gamma collider are underway and described in the box on the previous page.

The necessary research and development to make it real will consist primarily of work on laser development and on optical elements. Much of this work is "table top," that is, it does not require a high energy beam of electrons. However, to have adequate confidence in the concept (so as to be able to build it into an NLC), it will be necessary to develop a $y$ device working in the tens of $\mathrm{GeV}$ range. This is necessary in order to study backgrounds, detector issues (such as compatibility with the required optical elements), and lifetime of optical elements in a collision-point background.

\section{HIGGS TWO GAMMA WIDTH}

A gamma-gamma collider is uniquely suited for a direct measurement of the partial decay width of a Higgs boson into two gamma quanta. The decay amplitude involves loops of any charged particles whose mass is derived from the Higgs mechanism, as shown below.

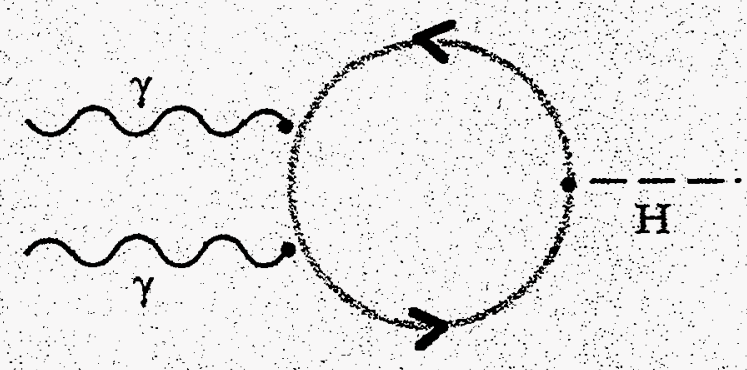

Feynman diagram for two photon decay of the Higgs boon. The loop can be of any charged elementary particles whose mass is generated via the Higgs mechanism.

A measurement of the two-photon width would be a senstive tes of various models predicting higher mass particles without producing them directly in accelerators. There are several such models with two photon couplings different from that of the standard model: super symmetric models, technicolor models, and other extensions of the Standard Models. 


\section{TECHNOLOGIES FOR CONSTRUCTING HIGH POWER LEASER}

High power diode lasers for pumping and lasing materials that can handle high thermal loading are two major efforts on advancing diode laser technologies already under way as part of both military and civilian projects. Advanced solid state materials, either athermal glass hosts or new crystals specifically engineered for diode pumping are also being actively developed. Based on these developments, the $16 \mathrm{~kW}$ laser needed for a gamma-gamma collider can bebuilt out of $1 \mathrm{~kW}$ unit cells, schematically illustrated below. There needs to be two such lasers, one for each opposing electron beam. If the laser pulses could be reused, for example by storing them in an optical cavity, the requirement on the average power could be significantly relaxed.

Another possibility is a free-electron laser (FEL), So far, FELs have generated neither the requisite peak power (gigawatt has been achieved so far) nor average power (a few watts is the present situation). On the other hand, they have considerable promise The chirped pulseamplification technique can be applied in an induction-linac-based free electron laser to produce optical pulses of the required charaterisitics for a gamma-gamma collider as shown in the bottom figure.
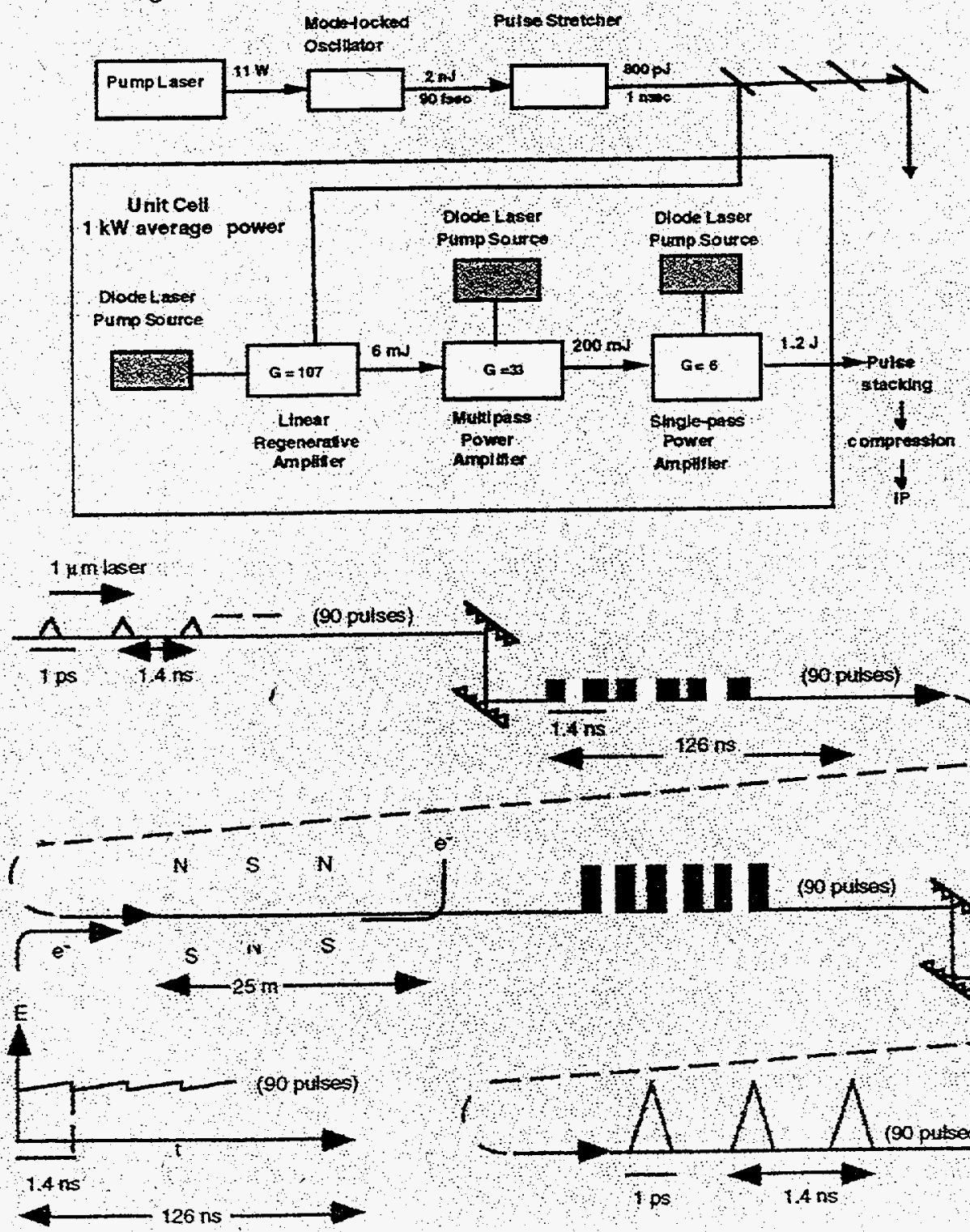

Left: A solid-state laser concept for the gamma-gamma collider built of $1 \mathrm{~kW}$ unit cells All of the cells are fed by a single phase-stabilized oscillator, ensuring synchronization of all pulses with the electron beam. Each of the unit cells consists of a series of diode-pumped, solid statelaser amplifiers. The pulses are subseguently compressed in a grating pair and stacked into a single pulse train

Left A free-electron laser that employs a pulse stretcher, an FEL amplifier, and a pulse compressor. 


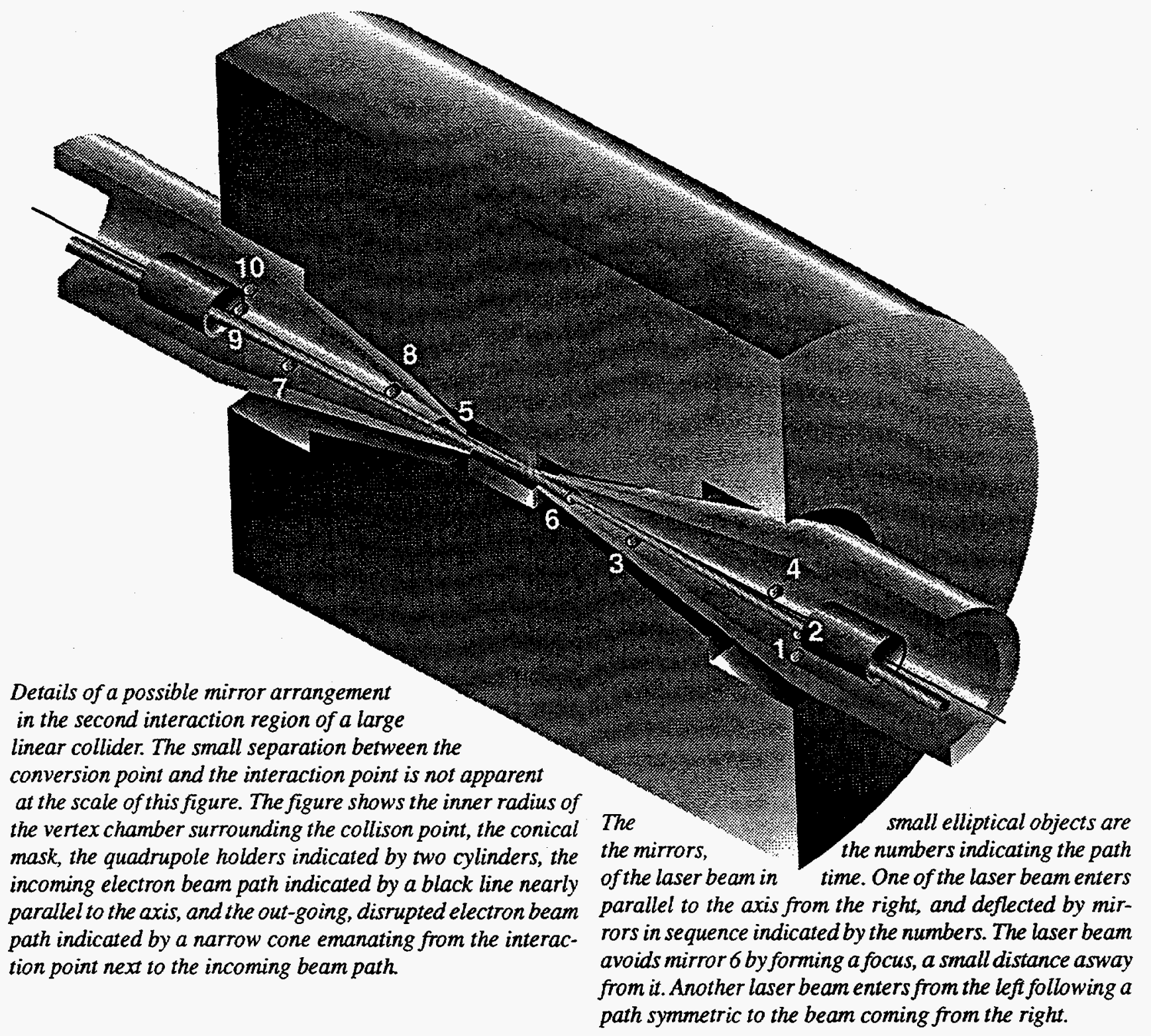

\section{The Prospects}

A very interesting possibility is the use of the SLAC Linear Collider (SLC), that is, extending the capability of the SLC (call it SLC II) to ye-collisions. Collisions of $\gamma e$ have a greater energy reach and thus are much more interesting, at the SLC, for particle physics than $\gamma \gamma$ collisions. Either, of course, is of interest for technological studies. One could study the spin asymmetry in the process $\gamma^{-} \rightarrow \mathrm{Ze}^{-}$, which tests the Standard Model and probes anomalous $\gamma \mathrm{ZZ}$ couplings, while energy scans near the threshold of $\gamma e^{-} \rightarrow W-v$ may lead to an improved $W$ mass measurement.

We have seen that a $\gamma$ collider is technically possible and that it would open up important new possibilities for particle physics research. Such a project will require a significant investment in preparatory research and development. Our hope is that the high energy community will come to realize the outstanding promise of the gamma-gamma collider idea and will respond by offering its participation and support. 